\section{Renin and aldosterone response in human newborns to acute change in blood volume}

Sir,

Referring to the paper by Dillon et al. (Archives, 1978, $53,461)$, we should like to report our studies on 26 term neonates, who were exchange transfused and compared with 10 healthy controls. The aetiology of jaundice of the exchanged newborns was Rhesus incompatibility in 5 cases, ABO incompatibility in 11, G-6-PD deficiency in 5 , and in the remaining 5 it could not be determined. The blood used for exchange transfusion was supplemented with citrate dextrose (ACD). Angiotensin I levels were measured, by the radioimmunoassay technique of Haber et al. (1969), immediately before, in the middle, and at the end of the exchange transfusion. The middle and end transfusion specimens were taken after a 10-min interruption of the exchange transfusion. No loading of the baby's circulation was made by leaving a surplus. Serum $\mathrm{Na}$, Hct, and $\mathrm{Hb}$ were also determined.

The angiotensin I levels of the exchange transfused infants at the beginning $(1 \cdot 36 \pm 0 \cdot 18 \mathrm{SE} \mathrm{ng} / \mathrm{ml}$ per hour), the middle $(1 \cdot 75 \pm 0 \cdot 18)$, and the end of the exchange transfusion $(1.47=0.18)$ did not differ significantly, although an increase was observed in the middle. Neither did the serum sodium levels differ.

The mean level $\pm S E$ of angiotensin in the blood used for the exchange transfusion was $0 \cdot 84 \pm 0 \cdot 13$. If the aetiology of jaundice is taken into consideration, the angiotensin I levels of the infants, whose jaundice was due to G-6-PD deficiency, were lower $(0.87 \pm 0.23)$ at the beginning of the exchange transfusion than those of infants with jaundice due to Rhesus incompatibility $(1 \cdot 36 \pm 0 \cdot 46), \mathrm{ABO}$ incompatibility $(1 \cdot 52 \pm 0 \cdot 27)$, or to unknown causes $(1.47 \pm 0 \cdot 30)$, or to the levels of the healthy controls $(1 \cdot 59 \pm 0 \cdot 16)$. No correlation between the Hct and angiotensin levels was noted. Lower levels of angiotensin I were found before the exchange transfusion in infants on their first 2 days of life.

From the above data we can conclude that exchange transfusion, if done slowly and lasting $1 \frac{1}{2}-2$ hours, does not influence the angiotensin levels of the newborn. Moreover this slow rate of exchange transfusion corresponds to the one (of $5 \mathrm{ml} / \mathrm{kg}$ per $3 \mathrm{~min}$ ) recommended by Aranda and Sweet (1977) as producing only minimal, rapid, reversible changes in the blood pressure. The observed lower angiotensin I levels in the jaundiced infants with G-6-PD were probably due to the young age ( $2 \frac{1}{2}$ days median) of the infants and not to increased haemolysis.

S. Frangatos, E. Hadjigeorgiou, D. Alexiou, C. Constantinides, N. Kalpoyiannis, and D. Nicolopoulos Department of Clinical Therapeutics of Athens University, and Neonatal Department, Alexandra Maternity Hospital, Athens 611, Greece

\section{References}

Aranda, J. V., and Sweet, A. Y. (1977). Alterations in blood pressure during exchange transfusion. Archives of Disease in Childhood, 52, 545-548.
Haber, W., Koerner, T., Page, L. B., Kliman, B., and Purnode, A. (1969). Application of a radioimmunoassay for angiotensin I to the physiologic measurements of plasma renin activity in normal human subjects. Journal of Clinical Endocrinology and Metabolism, 29, 1349-1355.

\section{Gluten challenge in treated coeliac disease}

Sir, In their interesting paper (Archives, 1978, 53, 449), Packer et al. state that (after a 3- to 4-month period of gluten ingestion) 'the finding of a normal postchallenge biopsy excludes coeliac disease', but they also counsel that such children should be followed for up to 2 years after challenge, with the implication that a normal biopsy at this time definitely excludes coeliac disease.

We have made a preliminary report of a similar study which is being published in greater detail (McNicholl et al., 1979). 40 children were challenged with gluten after a mean of 5.8 years on gluten-free diets and so far 37 have been confirmed to have coeliac disease by the occurrence of mucosal relapse. In some children, the mucosal relapse was very much slower than in the patients of Packer et al. In one girl after 28 months on gluten the overall appearance of the mucosa was just acceptable as normal and the interepithelial lymphocytes and mucosal disaccharidases were normal; it was not until the 63rd month on gluten that the mucosa was sufficiently abnormal to indicate definite relapse. Throughout this period she showed no haematological abnormalities and remained on the 75th centiles for height and weight.

We strongly reinforce the recommendation of Packer et al. that coeliac disease should not be diagnosed without satisfactory biopsy and agree that it was necessary for them to attempt to prove or disprove the initial diagnosis of coeliac disease (probably made by others) by gluten challenge. However, we would regard the 6 children with normal biopsies after 2 years on gluten as unsatisfactory subjects for allowing conclusions to be drawn concerning gluten intolerance, in the absence of an initial diagnostic biopsy. We agree with them that there are no consistent biochemical, haematological, or anthropometric indices of mucosal relapse. Finally, our experience dictates (Egan-Mitchell et al., 1978) that children whose initial mucosa had been definitely abnormal and who had fulfilled other accepted criteria for coeliac disease, must be followed up for considerably longer than 2 years, to allow a primary diagnosis of coeliac disease to be discarded.

B. MCNicholl, B. EgaN-Mitchell, and P. F. FotTRELL Department of Paediatrics, Regional Hospital, Galway, Eiré 